\title{
A comparison of verification in the temporal and cepstrum-transformed domains of Transient Evoked Otoacoustic Emissions for biometric identification
}

\section{Paul Chambers, Neil J. Grabham and Matthew A. Swabey}

School of Electronics and Computer Science, University of Southampton, Southampton SO17 1BJ, UK E-mail: pc@ecs.soton.ac.uk E-mail:njg@ecs.soton.ac.uk E-mail: mas@ecs.soton.ac.uk

\section{Mark E. Lutman}

Institute of Sound and Vibration Research, University of Southampton, Southampton SO17 1BJ, UK E-mail: mel@isvr.soton.ac.uk

\section{Neil M. White}

School of Electronics and Computer Science, University of Southampton, Southampton SO17 1BJ, UK

E-mail: nmw@ecs.soton.ac.uk

\section{John E. Chad}

School of Biological Sciences,

University of Southampton,

Southampton SO17 1BJ, UK

E-mail: jchad@soton.ac.uk

\section{Stephen P. Beeby*}

School of Electronics and Computer Science, University of Southampton, Southampton SO17 1BJ, UK E-mail: spb@ecs.soton.ac.uk ${ }^{*}$ Corresponding author 


\begin{abstract}
An investigation of methods for the use of Transient Evoked Otoacoustic Emissions (TEOAE) as an identification biometric is described in this paper. Three methods to calculate the Euclidean distance between individuals are investigated: Interpretation of area-under-the-curve information from time-series TEOAE; Measurement in the temporal domain between a TEOAE and reference template; Measurement in the cepstral domain between a TEOAE and reference template. False acception and rejection rates (FAR\&FRR) are given for test data. In this study maximal performance was given by the temporal domain Euclidian distance, giving $1.27 \% \mathrm{FAR}$ and $0 \%$ FRR for a data set with 230 recordings from 23 subjects.
\end{abstract}

Keywords: biometric identification; otoacoustic emissions; ear; biometrics; security; identification; verification.

Reference to this paper should be made as follows: Chambers, P., Grabham, N.J., Swabey, M.A., Lutman, M.E., White, N.M., Chad, J.E. and Beeby, S.P. (2011) 'A comparison of verification in the temporal and cepstrum-transformed domains of Transient Evoked Otoacoustic Emissions for biometric identification', Int. J. Biometrics, Vol. 3, No. 3, pp.246-264.

Biographical notes: Paul Chambers graduated with a BEng (Hons) in Electronic Engineering from the University of Limerick, Ireland, in 2001 and completed a PhD in Optoelectronic Gas Detection at the Optoelectronics Research Centre (ORC) at the University of Southampton in 2005. This was followed by a two-year IRCSET research fellowship studying optical gas detection at the University of Limerick.

Neil J. Grabham is a Senior Research Fellow in the Electronic Systems and Devices group in the School of Electronics and Computer Science at the University of Southampton. He holds an MEng (Merit) in Information Engineering and a PhD for a thesis titled 'Development of a Thick-Film Magnetostrictive Material: Formulation And Characterisation' from the University of Southampton, awarded in 1998 and 2002, respectively. His current areas of research cover sensor technology, biometrics, energy harvesting and wireless sensor networks. He is a member of the Institution of Engineering and Technology and a member of the Institute of Physics.

Matthew A. Swabey received his BEng (Hons) in Electronic Engineering and his $\mathrm{PhD}$ in Otoacoustic-based Biometrics from the University of Southampton, UK, in 2001 and 2005, respectively. After active research following the subject of his $\mathrm{PhD}$, he was appointed as a Teaching Fellow with the School of Electronics and Computer Science, Southampton University, UK, and currently pioneers novel microelectronics courses in high-level system on chip design. $\mathrm{He}$ is a member of the IET.

Mark E. Lutman is Head of the Hearing and Balance Centre at the Institute of Sound and Vibration Research of the University of Southampton. He joined ISVR in 1995 as Professor of Audiology from the Medical Research Council's Institute of Hearing Research in Nottingham. He is an Audiologist with main interests in research and teaching. His research interests include measurement of cochlear function from otoacoustic emissions, neonatal hearing screening, cochlear implantation, psychoacoustics, epidemiology of hearing impairment, evaluation of benefit from hearing instruments and noise-induced hearing loss. He was President of the British Academy of Audiology from 2007 to 2008 . 
Neil M. White is Head of the Electronic Systems and Devices Group and Deputy Head of School (Enterprise) within the School of Electronics and Computer Science. He is a co-founder and former Director of Perpetuum Ltd., a University energy harvesting-based spin-out company. He has over 200 publications in the area of sensor technology. He is a Chartered Engineer and Physicist, Fellow of the Institution of Engineering and Technology, Fellow of the Institute of Physics and Senior Member of the IEEE.

John E. Chad is a Senior Lecturer in Biological Sciences at the University of Southampton, UK, and a member of the Southampton Neurosciences Group. In 2002, he co-founded Capsant Neurotechnologies Ltd., which is conducting research into neurodegenerative diseases. His background includes research as a Faculty Research Biologist at UCLA (1978-1985) studying neuronal calcium signalling, and research at the Sandoz Institute for Medical Research (1985-1986). His research interests include the application of optics to neuroscience, neurocomputation, control of neuronal excitability and neurodegeneration.

Stephen P. Beeby obtained a PhD in Micromechanical Resonators from the University of Southampton in 1998. He was awarded a prestigious EPSRC Advanced Research Fellowship in 2001 and is currently a Reader in the School of Electronics and Computer Science at the University of Southampton. His research interests include biometrics, microsystems and energy harvesting. $\mathrm{He}$ is the coordinator of an EU Framework Integrated Project 'MICROFLEX' and is principal or co-investigator on further three projects. He has co-authored one book, MEMS Mechanical Sensors, published over 135 publications in the field and has 5 patents to his credit.

\section{Introduction}

Whilst it well known that ears receive and process sounds, which are sensed by hair cells located in the cochlea in the inner ear, it is not as widely known that there is also an active process occurring in the cochlea, which adds energy to incoming sound waves. The purpose of this is believed to be in order to overcome losses that occur due to damping. Energy is added by the outer hair cells, which can act as actuators when stimulated by deflection. This active process enables the ear to sense sounds, which would otherwise be too low level to detect. A by-product of this active process is the re-emission of sound from the ear, which results from the cochlea 'losing control' of a small amount of energy. These emitted sounds are termed OAEs and were originally theorised in 1948 by Gold (1948); however, they were not experimentally measured until 1978 with the ground-breaking work of Kemp (1978). Kemp had experimentally measured a sound signal emanating from the ear cavity in response to a click stimulus. This signal is sufficiently complex that it has not been fully explained and accounted for to this date.

Given the existence of an individual marker of this complexity, it is more likely that these signals are indicative of the person's true identity, i.e., an individual's emissions are unique to that individual. For example, differences in the frequency and number of spontaneous OAEs have been previously observed between the genders (Bilger et al., 1990) and different ethnic backgrounds (Whitehead et al., 1993). This paper will extend the work of Swabey et al. (2009) who devised a biometric analysis based on time-series 
TEOAE data, and will compare this temporal domain analysis with one in the cepstral domain.

The signal that Kemp had measured in response to a click is now termed a TEOAE. The TEOAE is now one of a family of methods of recording OAEs, which are (Robinette and Glattke, 1997):

1 Transient Evoked Otoacoustic Emission (TEOAE): Complex sounds emitted from the ear canal several milliseconds after a very short click stimulus has been sounded into the ear canal; these emissions are due to the complicated process of the cochlea receiving sound. These sounds are termed 'cochlear echoes'.

2 Spontaneous Otoacoustic Emission (SOAE): Continuously emitted tones that can be detected from around 30\% (Robinette and Glattke, 1997) of young healthy ears without any stimulus.

3 Stimulus Frequency Otoacoustic Emission (SFOAE): These are emissions resulting from the application of a single frequency stimulus and are the result of reflections of travelling waves through the cochlea to the middle ear, where they create an audible emission adding to the sound energy in the ear canal.

4 Distortion Product Otoacoustic Emission (DPOAE): The sounds emitted from the ear in response to a stimulus comprising two tones, with differing frequency.

This is caused by the mechanical non-linearity of the outer hair cells within the cochlea (Knight and Kemp, 2000) whilst being stimulated by both frequencies.

This cellular non-linear emission process does not depend on any neural activity. Five TEOAEs, recorded from five different subjects (referred to as the Longitudinal Adult TEOAE Corpus) are shown in Figure 1. A simple visual comparison shows that there is a clear difference between these individuals TEOAEs. These emissions were recorded by the authors using an Otodynamics ILO292 system with a UGD TE+DPOAE probe and ILO V6 clinical software; due to subject confidentiality required by the authors' institution, this data set is not publicly available.

TEOAEs are recognised clinically as being unique and indicative of the status of an individual's hearing function (Cope and Lutman, 1988; Grandori and Ravazzani, 1993; Lutman et al., 1997; Vohr et al., 1998). The TEOAE is the most frequently used method of clinically evaluating OAEs and there are several commercially available products that measure the TEOAE. Because of its widespread use and robust nature, the TEOAE method is utilised in this investigation. The methods employed for clinically evaluating OAEs involve an evaluation of the time-series and the frequency range of the Fourier transform data, which has been shown to identify hearing defects with individuals (Robinette and Glattke, 1997).

For application as a biometric, it is essential that an individual's TEOAE is unique to that individual and can be clearly distinguished from any other individual. In biometrics literature, this uniqueness is quantified in terms of the FAR, which is the proportion of fraudulent users passed as valid and a FRR, which is the proportion of valid users incorrectly rejected. Another metric used to compare the performance of biometrics is the Equal Error Rate (EER), which is the percentage error at which the FAR and FRR are equal. In all these metrics, a lower percentage value is considered better performance. Within a particular application, a higher FAR may be accepted to ensure a lower FRR or 
vice versa. For example in a high security access scenario, the FAR may be required to be zero, whilst false rejections can be dealt with by the user providing additional forms of identification to a security guard.

Figure 1 A selection of TEOAEs from five individuals (see online version for colours)
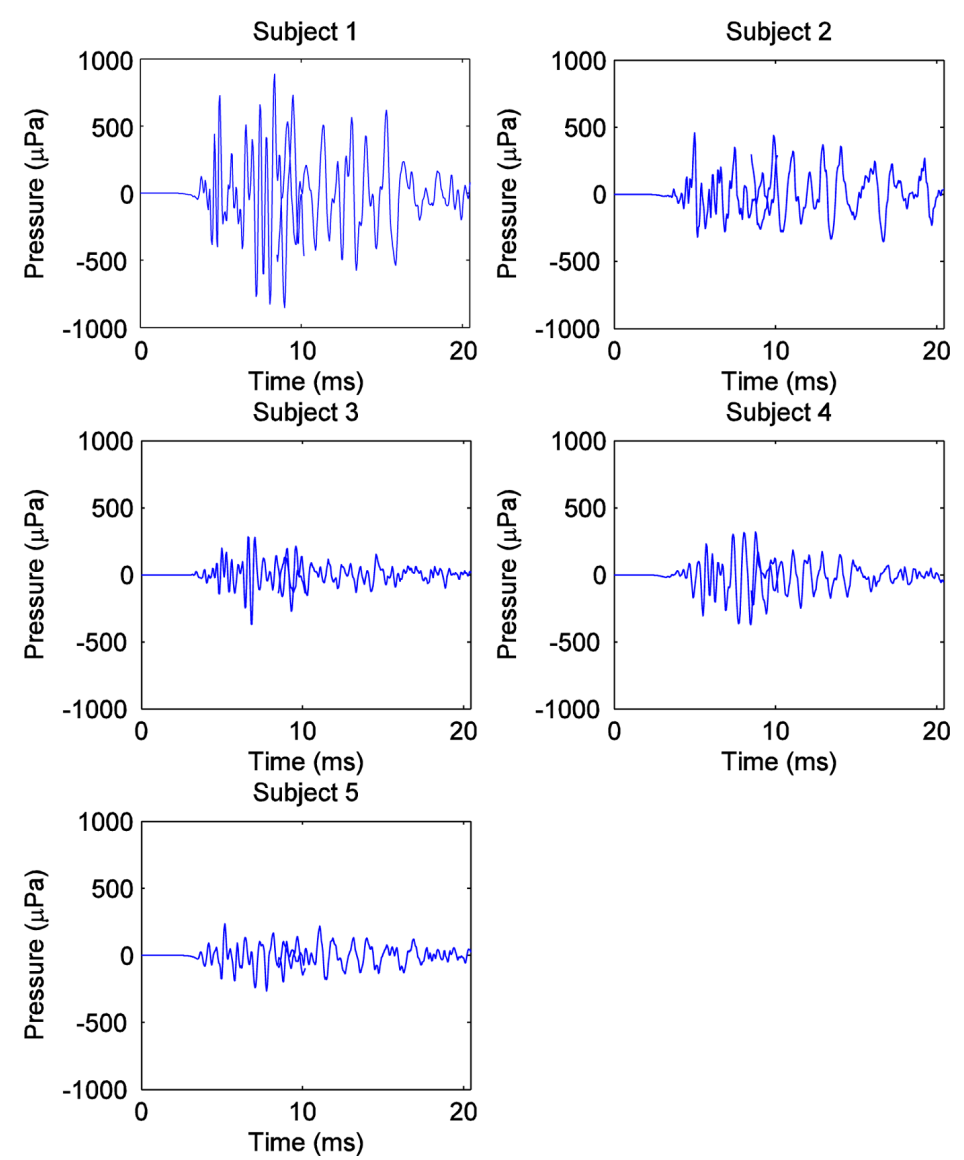

For the purpose of using a person's TEOAE as a biometric identifier as previously reported by Swabey et al. (2004), this paper investigates the following three techniques:

1 interpretation of the area-under-the-curve information from the time-series TEOAE data.

2 a measurement of the distance between a TEOAE recording in the temporal domain and a user template based on averaging prior recorded data

3 a measurement of the distance between a TEOAE recording transformed into the cepstral domain and a user template based on averaging cepstral-transformed prior recorded data.

To test the verification algorithms, two sets of recorded TEOAEs will be used. The first is the Longitudinal Adult TEOAE corpus, which is a data set consisting of multiple recordings of emissions from five adults recorded periodically over time. A second data 
set, the Transverse Adult TEOAE corpus, consists of recordings from 23 subjects, with 10 recordings being made for each subject at a single sitting. The purpose of the longitudinal corpus is to allow for investigation of any change in effect over time, whilst the transverse corpus allows for testing of uniqueness across a larger population. All the TEOAE recordings used have been made using commercially available equipment under controlled conditions.

\section{The TEOAE}

Five typical complex TEOAE responses to the same stimulus are shown in Figure 1. A TEOAE is a measurement of the response of the cochlea to an audio stimulus based on a click. This stimulus covers a range of frequencies due to the nature of the click. The time trace of a sample stimulus is shown in Figure 2. It can be observed that the stimulus is well defined and typically has a duration of approximately $1 \mathrm{~ms}$.

Figure 2 A sample stimulus used to evoke a TEOAE (see online version for colours)

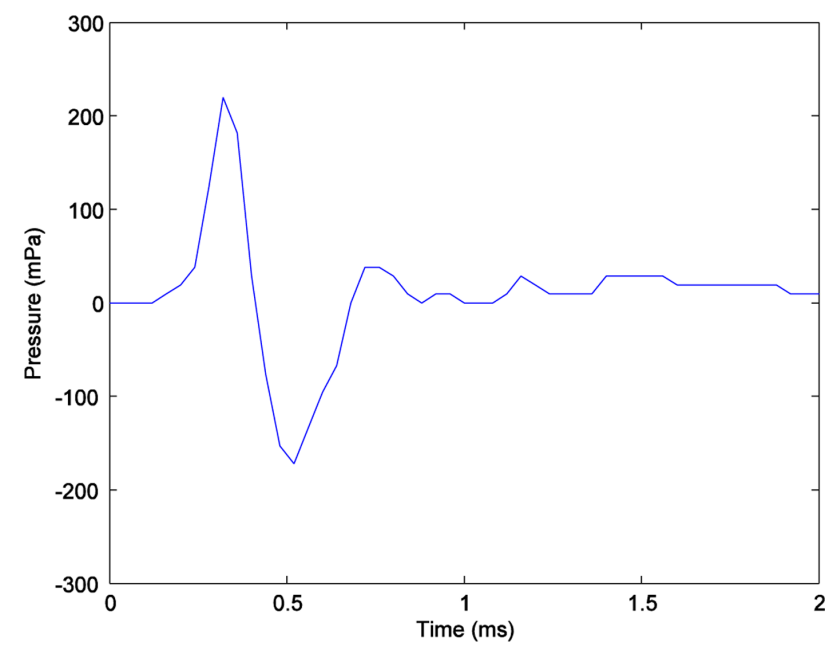

\section{Area-under-the-curve analyses of TEOAEs}

To make an initial illustrative analytic demonstration of the biometric markers that are contained within a TEOAE, the area enclosed by the magnitude of the time trace of the TEOAE has been calculated and the resulting area values for the Longitudinal Adult TEOAE corpus are evaluated.

The area values are determined from the time-series data of each TEOAE measurement using the formula shown in equation (1).

$$
A=p \cdot \sum_{t}|x(t)|
$$

where $x(t)$ are the time-series data-points, $p$ is the duration of each measurement period, and $A$ is the resulting enclosed area. The area data from 10 independent TEOAE 
measurements from each of five subjects from the Longitudinal Adult TEOAE Corpus have been calculated using equation (1), and the results are shown in Figure 3. Each TEOAE has 512 data points sampled at a rate of $25 \mathrm{kHz}$, providing a recording period of $20.48 \mathrm{~ms}$, which is sufficient to capture the full response from the stimulus.

Figure 310 TEOAE area measurements from 5 subjects (Recordings 1-10 are Subject 1, 11-20 Subject 2, 21-30 Subject 3, 31-40 Subject 4, 41-50 Subject 5) (see online version for colours)

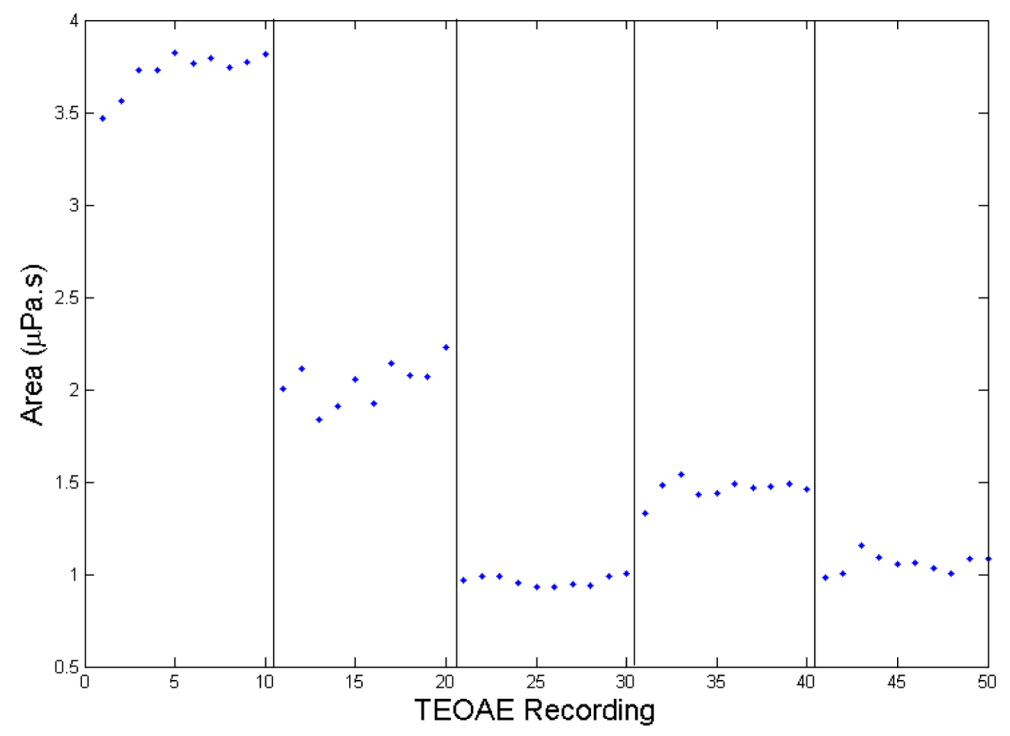

In Figure 3, the first 10 data points relate to Subject 1, the second 10 data points to Subject 2, and so forth. Each data point is obtained from a separate recording of a TEOAE. This fundamental area data is sufficient to show that there is a relatively small difference on an intra-user basis, while there is a typically larger difference on an inter-user basis. The value determined for the area under the emission magnitude graph is a measure of the level of the emission of the subject and as such does not distinguish between subjects based on the time response, but only as a function of the level of the overall response. The level of this response would be affected by the fit of the probe in the ear and variation in the quantity and nature of any sound damping matter within the ear canal such as ear wax. The inter-subject difference that is present does, however, suggest that the TEOAE does possess potential for use as a biometric. However, to obtain a larger inter-user difference, more sophisticated analysis must be used to enable maximum separation of different individuals TEOAEs. Two potential techniques, which can be used to increase the subject differentiation, are described in the following sections.

\section{Euclidean distance measure of the time-series data of a TEOAE}

The time-series data is probably the most easily visualised, as it is the signal dealt with by practitioners working in the field of otoacoustics. The time-series Euclidean 
distance measure is obtained by obtaining the root square of the summation of the difference of each element of the biphasic time-series data of two TEOAEs, as shown by equation (2):

$$
e t=\sqrt{\sum\left((m(t)-n(t))^{2}\right.}
$$

where $e t$ is the time-series Euclidean distance, $t$ is the time index, $m(t)$ represents the biphasic TEOAE recording, and $n(t)$ represents a second, template, biphasic TEOAE recording. Each TEOAE has 512 data points sampled at a rate of $25 \mathrm{kHz}$, providing a recording period of $20.48 \mathrm{~ms}$, which is sufficient to capture the full response from the stimulus.

The following analysis uses a template formed by averaging seven TEOAEs, which is then compared with further TEOAEs for each individual. Subsequent TEOAE recordings are compared with each template, with a good match being indicated by a small difference between the Euclidean distances. All the TEOAEs used for these tests are drawn from the Longitudinal TEOAE Adult Corpus.

In Figure 4, a template for Subject 1 has been compared against 10 other recorded TEOAEs from each of Subject 1 and four other subjects. The analysis is further demonstrated in Figure 5 where the reference TEOAE template is taken from Subject 3. It is clear the Euclidean distance measure is at a minimum when analysed on an intra-user basis and at a significantly higher value when analysed on an inter-user basis.

Figure 4 Time-series Euclidean distance of TEOAEs from five subjects compared against a reference TEOAE template from Subject 1. (Recordings 1-10 are Subject 1, 11-20 Subject 2, 21-30 Subject 3, 31-40 Subject 4, 41-50 Subject 5) (see online version for colours)

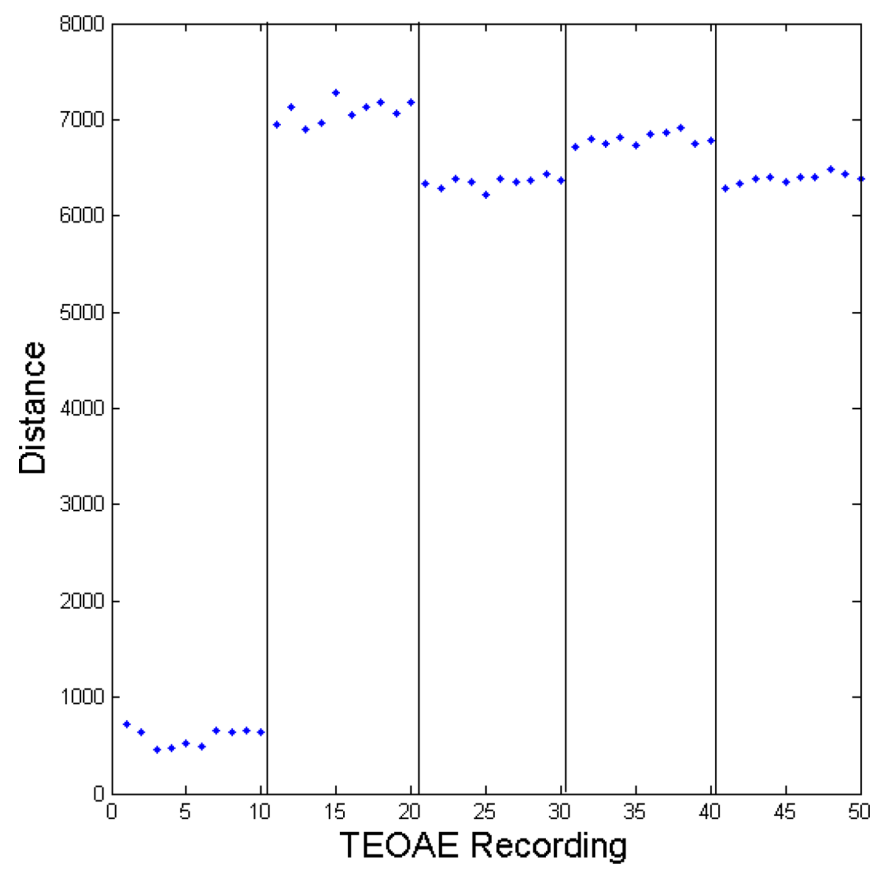


Figure 5 Time-series Euclidean distance of TEOAEs from five subjects compared against a reference TEOAE template from Subject 3. (Recordings 1-10 are Subject 1, 11-20 Subject 2, 21-30 Subject 3,31-40 Subject 4, 41-50 Subject 5) (see online version for colours)

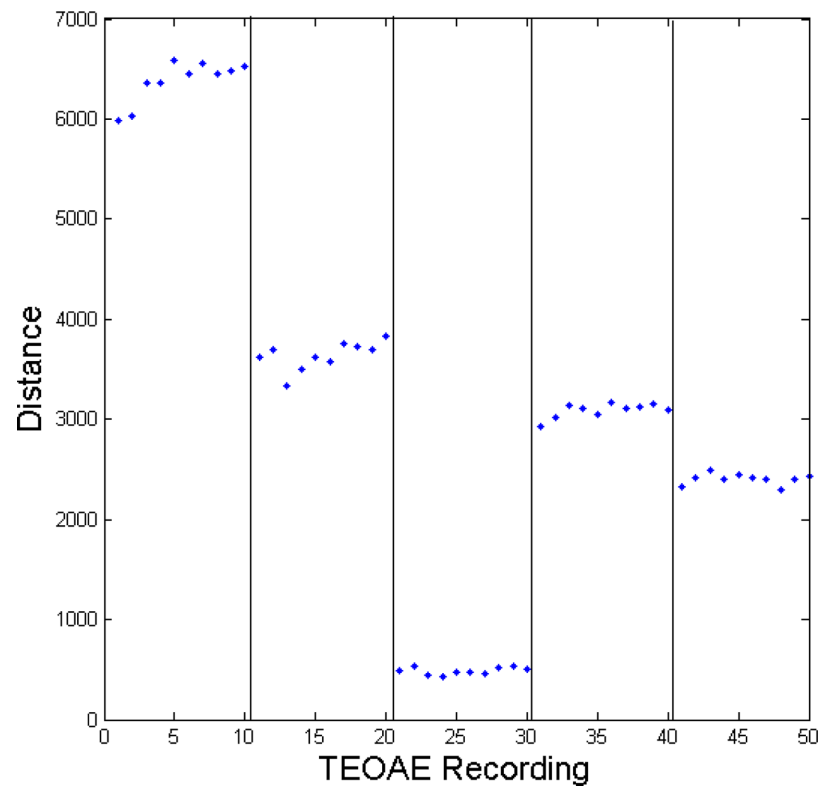

A mean distance is calculated for a reference template when compared with 10 further TEOAE recordings from the same subject using the formula shown as equation (2), and a standard deviation for these distances calculated. This process is first repeated for 10 TEOAE recordings from each of the other four subjects, and the results tabulated. The whole process is then repeated using a reference template from each of the other subjects in turn. The mean values and standard deviations for both the intra- and inter-subject tests for the five subjects analysed are shown in Table 1, with the intra-subject results shaded in the table. It can be seen that, in terms of the mean values obtained, each user is clearly distinct, i.e., that the mean values are at a minimum when users are compared on an intra-user basis and are significantly greater when users are compared on an inter-user basis.

Table 1 Inter- and intra-user mean and standard deviation (in brackets) of time-series Euclidean distances within the longitudinal adult TEOAE corpus (to calculate these mean and standard deviation values 10 separate TEOAE recordings have been used for each test subject)

\begin{tabular}{|c|c|c|c|c|c|c|}
\hline & & \multicolumn{5}{|c|}{ Reference subject } \\
\hline & & Subject 1 & Subject 2 & Subject 3 & Subject 4 & Subject 5 \\
\hline $\begin{array}{l}\frac{\overrightarrow{0}}{0} \\
\frac{.}{3}\end{array}$ & Subject 1 & $\begin{array}{l}589.65 \\
(92.97)\end{array}$ & $\begin{array}{c}7.03 \times 10^{3} \\
(231.68)\end{array}$ & $\begin{array}{c}6.37 \times 10^{3} \\
(209.07)\end{array}$ & $\begin{array}{c}6.81 \times 10^{3} \\
(213.89)\end{array}$ & $\begin{array}{r}6.40 \times 10^{3} \\
(208.12)\end{array}$ \\
\hline$\stackrel{\substack{\tilde{n} \\
\Leftrightarrow}}{\Leftrightarrow}$ & Subject 2 & $\begin{array}{c}7.07 \times 10^{3} \\
(119.81)\end{array}$ & $\begin{array}{c}1.35 \times 10^{3} \\
(374.07)\end{array}$ & $\begin{array}{c}3.63 \times 10^{3} \\
(141.44)\end{array}$ & $\begin{array}{c}3.80 \times 10^{3} \\
(132.98)\end{array}$ & $\begin{array}{c}3.54 \times 10^{3} \\
(137.79)\end{array}$ \\
\hline
\end{tabular}


Table 1 Inter- and intra-user mean and standard deviation (in brackets) of time-series Euclidean distances within the longitudinal adult TEOAE corpus (to calculate these mean and standard deviation values 10 separate TEOAE recordings have been used for each test subject) (continued)

\begin{tabular}{|c|c|c|c|c|c|c|}
\hline & & \multicolumn{5}{|c|}{ Reference subject } \\
\hline & & Subject 1 & Subject 2 & Subject 3 & Subject 4 & Subject 5 \\
\hline \multirow{3}{*}{ 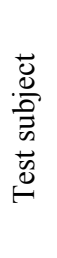 } & Subject 3 & $\begin{array}{c}6.34 \times 10^{3} \\
(59.43)\end{array}$ & $\begin{array}{c}3.42 \times 10^{3} \\
(32.17)\end{array}$ & $\begin{array}{l}485.93 \\
(35.17)\end{array}$ & $\begin{array}{c}3.05 \times 10^{3} \\
(29.03)\end{array}$ & $\begin{array}{c}2.39 \times 10^{3} \\
(48.18)\end{array}$ \\
\hline & Subject 4 & $\begin{array}{c}6.79 \times 10^{3} \\
(64.54)\end{array}$ & $\begin{array}{c}3.67 \times 10^{3} \\
(95.83)\end{array}$ & $\begin{array}{c}3.69 \times 10^{3} \\
(73.55)\end{array}$ & $\begin{array}{l}696.82 \\
(57.12)\end{array}$ & $\begin{array}{c}2.36 \times 10^{3} \\
(89.41)\end{array}$ \\
\hline & Subject 5 & $\begin{array}{c}6.38 \times 10^{3} \\
(53.68)\end{array}$ & $\begin{array}{c}3.38 \times 10^{3} \\
(58.58)\end{array}$ & $\begin{array}{c}2.40 \times 10^{3} \\
(55.53)\end{array}$ & $\begin{array}{c}2.32 \times 10^{3} \\
(72.93)\end{array}$ & $\begin{array}{l}608.73 \\
(57.53)\end{array}$ \\
\hline
\end{tabular}

The data in Table 1 shows that the maximum intra-user mean Euclidean distance was $1.35 \times 10^{3}$, and on an inter-user basis the minimum value was found to be $2.32 \times 10^{3}$. This shows a clear spacing between the maximum value of the intra-user Euclidean distance dataset and minimum value of the inter-user Euclidean distance dataset. If we assume that the Euclidean distance results have a normal distribution, we can calculate 95\% Confidence Interval (CI) values for the Euclidean distances. In the case of the intra-user distance, the $95 \%$ CI upper limit is $2.08 \times 10^{3}$, and in the case of inter-user distance the $95 \%$ CI lower limit is $2.18 \times 10^{3}$, showing that the two closest subjects from the adult TEOAE corpus can still be reliably classified.

\section{Euclidean distance measure of the cepstrum series data of a TEOAE}

In addition to the utilisation of the time-series data of the TEOAE for biometric identification, the Euclidean distance technique has also been applied to the cepstrum-transformed temporal TEOAE data. This analysis was again performed on data from the adult TEOAE corpus. The cepstrum is a recognised method used for feature extraction in speech analysis and seismology (Charbuillet et al., 2009; Oppenheim and Schafer, 2004). This technique can be applied to extracting distinctive biometric marker components from a TEOAE.

The mathematical expression that performs the cepstrum transform is shown in equation (3):

$$
c(t)=\operatorname{abs}(\operatorname{FFT}(\log (\operatorname{FFT}(x(t)))))
$$

where $x(t)$ is the original TEOAE time series, $\operatorname{abs}()$ is the absolute value function, FFT( ) is the Fast Fourier transform, $\log ()$ is the complex logarithm and $c(t)$ is the resultant cepstrum-transformed time series. Once the TEOAE has been transformed by the cepstrum transform from the temporal domain, it can then be said to be in the cepstral domain. The fast Fourier transform is performed using MATLAB, with 512 data points in the transform.

Five cepstrum-transformed TEOAEs are shown in Figure 6, which were derived from the time traces shown in Figure 1. The cepstrum-transformed graphs show an initial large peak representing the signal strength and a series of smaller peaks thereafter representing the signal modulation of the complex TEOAE. 
Figure 6 Cepstrum-transformed TEOAEs from the five TEOAEs that were previously shown in Figure 1 (see online version for colours)
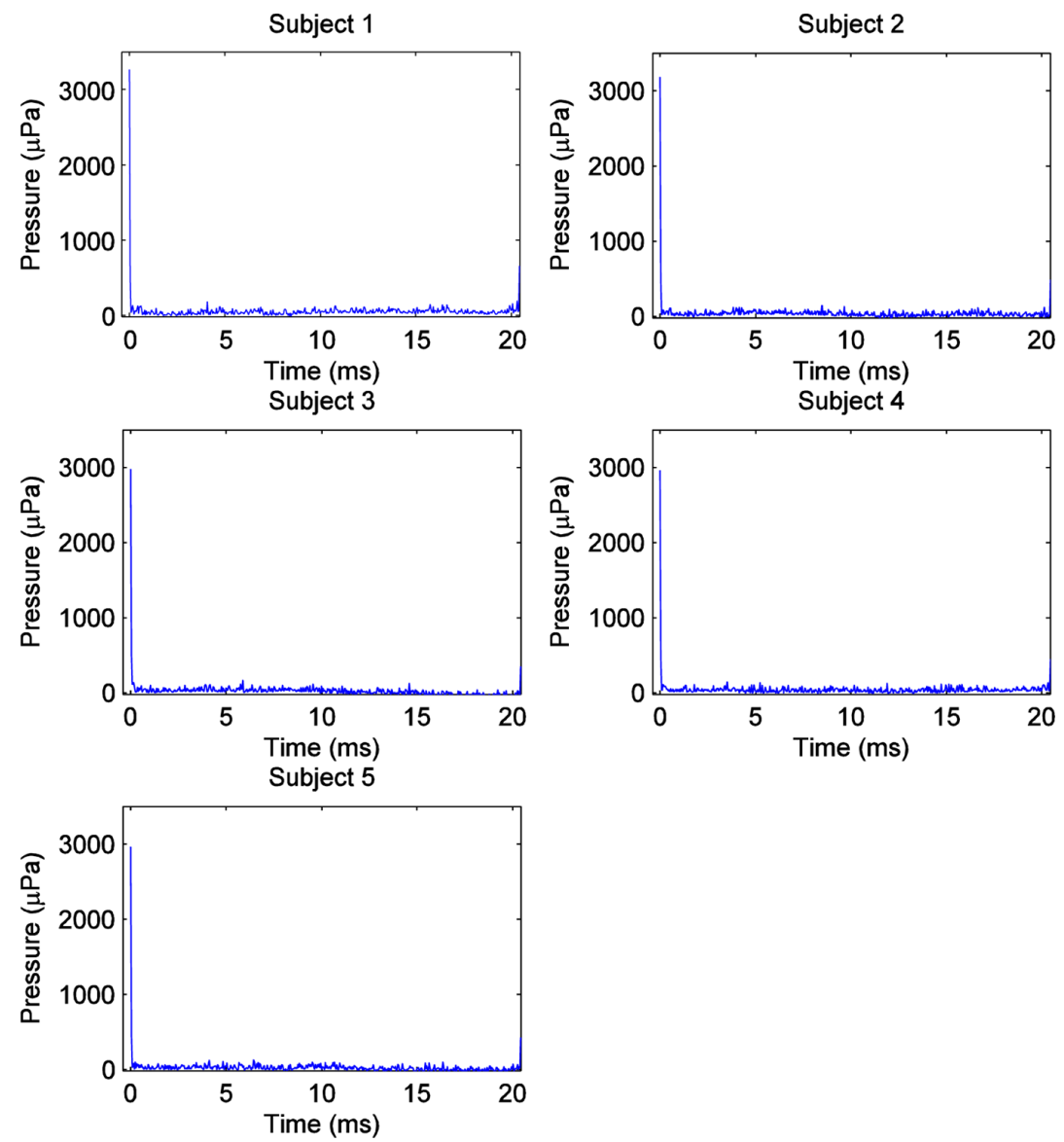

A Euclidean distance measure of the cepstrum-transformed TEOAE was investigated for application as a biometric. This is done in a similar manner to that shown in equation (2), except it now uses the cepstrum-transformed data as shown in equation (4):

$$
c t=\sqrt{\sum_{t}(m(t)-n(t))^{2}}
$$

where $c t$ is the cepstrum data Euclidean distance, $t$ is the time index, $m(t)$ represents the cepstrum-transformed TEOAE recording and $n(t)$ represents a second, template, cepstrum-transformed TEOAE recording. This analysis is performed using an identical methodology as that applied to the time-series Euclidean distance. Figure 7 shows a template-cepstrum-transformed TEOAE, again formed by averaging seven cepstrum transformed TEOAE recordings, from Subject 1 compared against 10 other recorded TEOAE cepstrum-transformed recordings from Subject 1 and a further 10 recordings from each of the four other subjects. Again, this analysis is performed on data from the Longitudinal TEOAE Adult Corpus. 
Figure 7 Cepstrum-transformed Euclidean distance of TEOAEs from five subjects compared against a reference TEOAE template from Subject 1 (Recordings 1-10 are Subject 1, 11-20 Subject 2, 21-30 Subject 3, 31-40 Subject 4, 41-50 Subject 5) (see online version for colours)

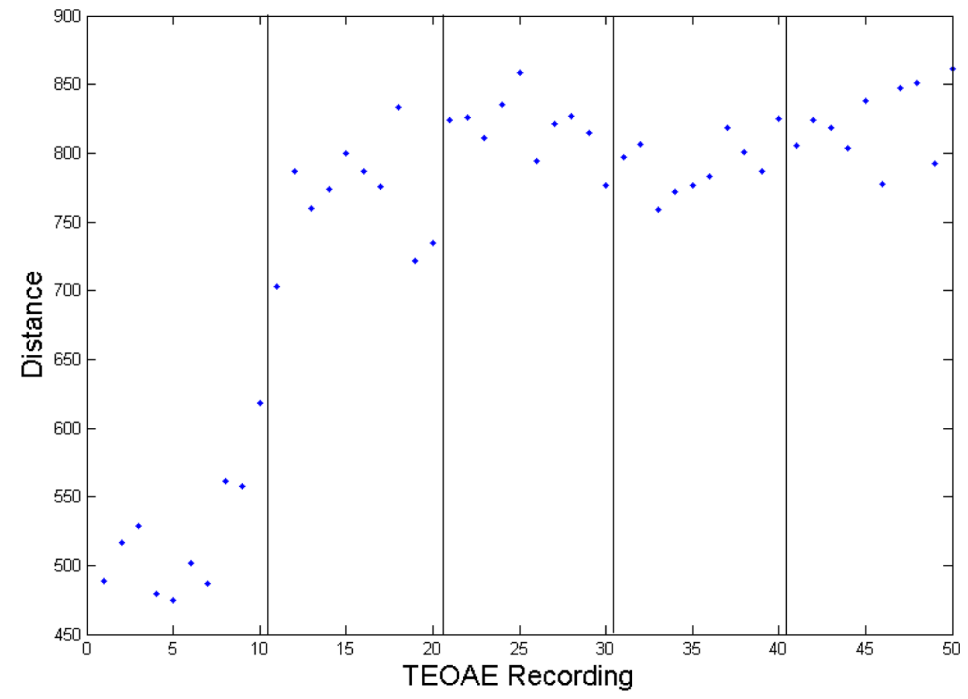

Figure 8 shows the results of repeating this analysis using a reference template from Subject 3. As with the time-series Euclidean distance measure, the cepstrum analysis can be seen by inspection to be at a minimum when analysed on an intra-user basis and at a significantly higher value when analysed on an inter-user basis.

Figure 8 Cepstrum-transformed Euclidean distance of TEOAEs from five subjects compared against a reference TEOAE template from Subject 3 (Recordings 1-10 are Subject 1, 11-20 Subject 2, 21-30 Subject 3, 31-40 Subject 4, 41-50 Subject 5) (see online version for colours)

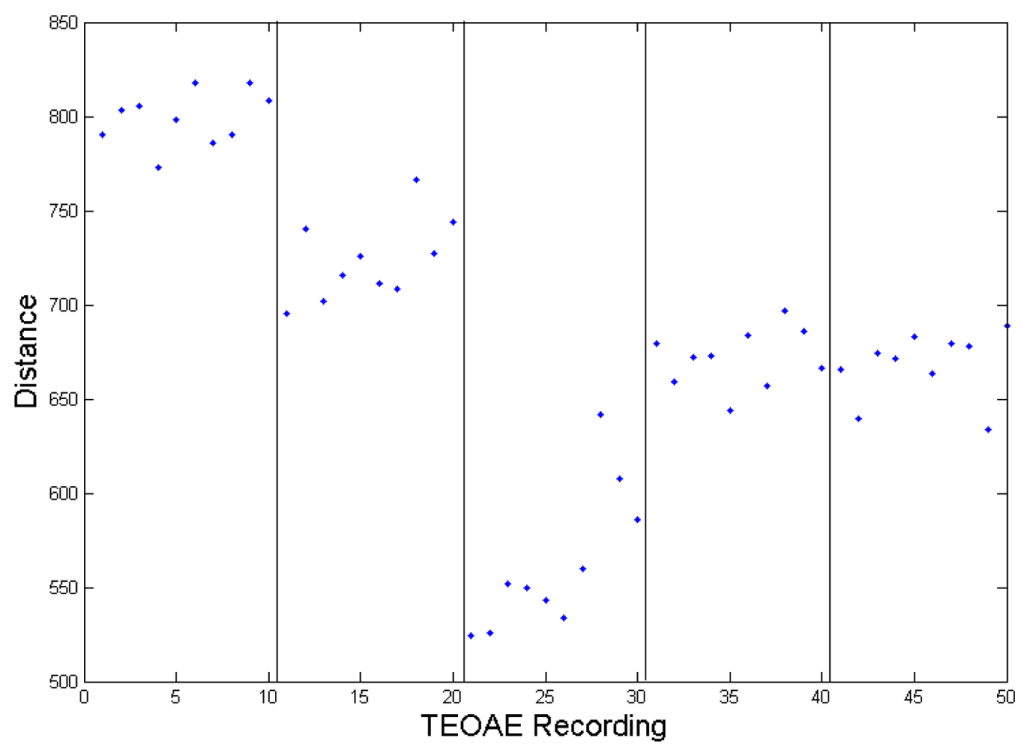


Table 2 shows the cepstrum mean values and standard deviation of the intra- and intersubject data relating to the adult corpus analysed where it can be seen that, in terms of the mean values obtained, each user is clearly distinct. It may be observed that the mean values are at a minimum when users are compared on an intra-user basis and are significantly greater when users are compared on an inter-user basis. If we assume that the Euclidean distance results have a normal distribution, we can calculate $95 \%$ CI values for the Euclidean distances. In the case of the intra-user distance, the $95 \%$ CI upper limit is 664.19, and in the case of inter-user distance the $95 \%$ CI lower limit is 610.22 , showing that the two closest subjects from the adult TEOAE corpus cannot be reliably classified. Taking a 90\% CI gives intra- and inter-user distances of 645.62 and 619.16, respectively, this shows that at the $90 \%$ CI the two closest subjects can again not be reliably classified. With the CI reduced to $75 \%$, the intra- and inter-user distances become 619.72 and 631.61 , at which the two closest subjects can be classified, this can be compared with the temporal domain tests where the closest subjects could be reliably classified at the $95 \% \mathrm{CI}$.

Table 2 Cepstrum-transformed Euclidean distance inter- and intra-user mean and standard deviation (in brackets) of the adult TEOAE corpus

\begin{tabular}{|c|c|c|c|c|c|c|}
\hline & & \multicolumn{5}{|c|}{ Reference subject } \\
\hline & & Subject 1 & Subject 2 & Subject 3 & Subject 4 & Subject 5 \\
\hline \multirow{5}{*}{ 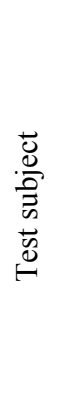 } & Subject 1 & $\begin{array}{l}551.41 \\
(45.7)\end{array}$ & $\begin{array}{l}746.57 \\
(15.21)\end{array}$ & $\begin{array}{l}798.93 \\
(14.40)\end{array}$ & $\begin{array}{l}757.09 \\
(17.01)\end{array}$ & $\begin{array}{l}788.00 \\
(12.98)\end{array}$ \\
\hline & Subject 2 & $\begin{array}{l}767.62 \\
(38.97)\end{array}$ & $\begin{array}{l}559.55 \\
(52.32)\end{array}$ & $\begin{array}{l}723.56 \\
(21.79)\end{array}$ & $\begin{array}{l}703.84 \\
(24.37)\end{array}$ & $\begin{array}{l}713.26 \\
(24.80)\end{array}$ \\
\hline & Subject 3 & $\begin{array}{l}818.91 \\
(22.33)\end{array}$ & $\begin{array}{l}724.80 \\
(31.37)\end{array}$ & $\begin{array}{l}562.40 \\
(38.12)\end{array}$ & $\begin{array}{l}660.56 \\
(25.17)\end{array}$ & $\begin{array}{l}661.30 \\
(22.59)\end{array}$ \\
\hline & Subject 4 & $\begin{array}{l}792.48 \\
(20.91)\end{array}$ & $\begin{array}{l}713.59 \\
(12.70)\end{array}$ & $\begin{array}{l}671.59 \\
(15.67)\end{array}$ & $\begin{array}{l}568.93 \\
(41.55)\end{array}$ & $\begin{array}{l}656.72 \\
(18.28)\end{array}$ \\
\hline & Subject 5 & $\begin{array}{l}792.48 \\
(27.31)\end{array}$ & $\begin{array}{l}721.80 \\
(14.24)\end{array}$ & $\begin{array}{l}667.72 \\
(18.15)\end{array}$ & $\begin{array}{l}657.73 \\
(17.66)\end{array}$ & $\begin{array}{l}568.90 \\
(45.49)\end{array}$ \\
\hline
\end{tabular}

As the levels of the first two coefficients in the cepstrum-transformed TEOAEs are of greater magnitude than the subsequent coefficients, as can be seen in Figure 6, a series of tests were performed to examine the effects of removing coefficients from the comparison. Of the 512 coefficients in the cepstrum-transformed TEOAE, selected ones were excluded from those used to calculate the Euclidean distance as described earlier in this section. The Euclidean distances are compared with a fixed threshold level to determine if the responses under test are to be classified as a match or not, the result of this test is then used to generate numbers of false rejects and accepts over the data set. To compare the effects of varying coefficients and ranges of coefficients on the overall efficacy of the verification process, the numbers of false rejects and accepts are compared in Table 3, in all cases the data set used is the Longitudinal TEOAE Adult Corpus.

Reviewing the results shown in Table 3 allows us to comment on the unique information contained in the different coefficients. First, it can be seen that excluding the first coefficient increases the number of false accepts by 10 but has no effect on the number of false rejects when compared with using all the coefficients, this implies 
that whilst the first coefficient does contain identification information it does not contain a high proportion of the overall identification information. Likewise excluding the second coefficient has a similar result. This is further reinforced by using just the first and second coefficients, which contain the peak in the response seen in the cepstrum-transformed TEOAEs shown in Figure 6, as can be seen from the table just using these coefficients significantly increases the number of errors. Further tests were performed to see if the majority of the information was carried in the lower order coefficients by using the first 10 and 100 coefficients, in both cases a significant difference in the number of errors is seen. In conclusion, it can be seen that unique identification information is contained throughout the Cepstrum coefficients and not just in the higher magnitude coefficients.

Table 3 Effect of removing cepstrum coefficients from Euclidean distance calculation on verification efficacy (based on a total of 512 cepstral coefficients)

\begin{tabular}{lcc}
\hline Cepstrum coefficients used & Number offalse rejects & Number of false accepts \\
\hline All coefficients & 1 & 47 \\
2nd to 512th & 1 & 57 \\
1st and 3rd to 512th & 1 & 49 \\
1st and 2nd & 23 & 422 \\
1st to 10th & 10 & 179 \\
1st to 100th & 11 & 162 \\
1st to 256th & 6 & 79 \\
1st, 2nd and 512th & 17 & 336 \\
\hline
\end{tabular}

\section{Comparison of the temporal domain and cepstral domain Euclidean distance measures for use as a biometric}

The analysis thus far has used the Longitudinal Adult TEOAE Corpus of five subjects to show the uniqueness of different individual's TEOAEs. To further test and compare the performance of the Euclidean distance algorithm within the temporal and cepstrum domains, the Transverse Adult TEOAE Corpus with its greater number of individual subjects has been used.

To compare the performance of the two domains, the same protocol as used by Swabey et al. (2009) has been used. This protocol builds on the work of Mansfield and Wayman (2002) who address evaluating the performance of a biometric with statistical tools where there is a lack of data. This lack of data is the typical case in biometrics as the goal is to completely separate the distributions of inter- and intra-users leaving no data in the gap between them where the PDF tails cross. The protocol consists of traditional statistical estimation along with numerical checks to ensure their validity:

- Form a reference template by averaging a set number of TEOAE recordings from one subject. The recordings used to form the template are subsequently excluded from the remainder of the testing process. 
- Calculate the intra- and inter-subject distances by comparing the individual TEOAE recordings against the template from the same subject and then against the templates from the other subjects. The number of intra- and inter-subject comparisons are given by:

Number of intra-subject comparisons $=n \times m$

Number of inter-subject comparisons $=n \times(n-1) \times m$

where $n$ is the number of subjects and $m$ is the number of TEOAE recordings remaining per subject after the template formation.

- Fit a Probability Density Function (PDF) to the intra- and inter-subject distances using Maximum Likelihood Estimation (MLE) (Severini, 2000) from the family of lognormal and normal continuous PDFs, respectively. The MLE process generates the maximally likely distribution parameters and the $95 \%$ CI relating to those parameters.

- Using the parameters from the MLE process calculate to a desired accuracy the crossover point of the distributions Cumulative Density Functions (CDFs) using a gradient descent algorithm. This value is the decision threshold that equalises the FAR and FRR, giving the EER, and is the Maximally Likely Threshold (MLT).

- Multiple distributions corresponding to the $95 \%$ CI per parameter and optimal parameter are calculated. We obtain four distributions for both the intra- and inter-subject distances, the maximum and minimum crossover values from these distributions are then used as the upper and lower thresholds that give a conservative estimate of the upper and lower 95\% confidence bounds on the MLE calculated EER.

- Other statistical and numerical results as defined in Table 4 are derived from the data set at the three values of the decision threshold as obtained from the MLE process to guarantee its credibility. By taking the observed FAR and FRR error rates, with bounds determined using the 'Rule of 30' or 'Rule of 3' as appropriate (Doddington et al., 2000), we can assess the quality of fit in the tail of the interand intra-user distance PDFs.

Table 4 Description of parameters used in comparison

\begin{tabular}{ll}
\hline Parameter & Definition \\
\hline Predicted EER & $\begin{array}{l}\text { EERs predicted by the crossovers of the: Lower 95\% confidence } \\
\text { interval CDF, Optimally fitted CDF, Upper 95\% confidence } \\
\text { interval CDF }\end{array}$ \\
Actual False Acceptances & $\begin{array}{l}\text { Actual number of false acceptances at each of the three MLE } \\
\text { thresholds }\end{array}$ \\
Actual False Rejections & $\begin{array}{l}\text { Actual number of false rejections at each of the three MLE } \\
\text { thresholds }\end{array}$ \\
& $\begin{array}{l}\text { FARs at the each of the three MLE thresholds, with confidences } \\
\text { FARs }\end{array}$ \\
\hline
\end{tabular}


Table 4 Description of parameters used in comparison (continued)

\begin{tabular}{ll}
\hline Parameter & Definition \\
\hline FRRs & FARs at the each of the three MLE thresholds, with confidences \\
& from the 'Rule of 30' or 'Rule of 3' as appropriate \\
& The $d^{\prime}$ scores for the three CDFs at the Lower 95\%, Optimal and \\
& Upper 95\% intervals
\end{tabular}

The verification process has been coded in MATLAB and has been applied to the Transverse Adult TEOAE Corpus, for the original temporal data and also to the cepstrum-transformed data. In the case of the cepstrum-transformed data, the transformation is performed prior to any other processing, such as forming the reference templates for each subject. Given that the Transverse Adult TEOAE Corpus used consists of 23 subjects, with 10 recordings per subject, and that three recordings per subject are used for template formation, there are 161 intra-subject comparisons and 3542 inter-subject comparisons in this analysis. Table 5 presents the resulting data from the statistical analysis for the two domains of interest for comparison, data is presented at the lower 95\% CI, the MLT, and at the upper 95\% CI. 'Rule of 30' CIs are given for the FARs, but due to the lower number of errors observed the FARs have a 'Rule of 3' bounding value.

Table 5 Comparison of performance of Euclidean distance in temporal and cepstral domains (values at lower 95\% CI/MLT/Upper 95\% CI)

\begin{tabular}{lcc}
\hline Parameter & Temporal domain & Cepstral domain \\
\hline Predicted EER & $0.469 / 1.17 / 2.59 \%$ & $1.04 / 2.02 / 3.61 \%$ \\
Actual FAR & $0.592 / 1.27 / 1.94 \%$ & $0.847 / 1.32 / 2.28 \%$ \\
Actual False Acceptances & $21 / 45 / 69$ & $30 / 47 / 81$ \\
Rule of 30 FAR Confidence & $\pm 50 / \pm 30 / \pm 30 \%$ & $\pm 30 / \pm 30 / \pm 30 \%$ \\
Actual FRR & $0.00 / 0.00 / 0.00 \%$ & $1.24 / 0.621 / 0.621 \%$ \\
Actual False Rejections & $0 / 0 / 0$ & $2 / 1 / 1$ \\
Rule of 3 FRR lower bound & $1.86 / 1.86 / 1.86 \%$ & $1.86 / 1.86 / 1.86 \%$ \\
$d^{\prime}$ & $8.89 / 8.55 / 8.19$ & $5.75 / 5.54 / 5.32$ \\
\hline
\end{tabular}

The values reported in Table 5 show that the performance of the Euclidean distance verification method used in this work performs better in the temporal domain than in the cepstral domain. This is shown by the lower predicted EER, calculated using the MLT analysis and then in the resulting false acceptance and rejection rates. To further compare the separation of the intra- and inter-subject distances, histograms of the distribution of distance values with fitted PDFs have been generated. These are shown as Figures 9 and 10, for the temporal and cepstral domains, respectively. Within these figures, the light grey histogram and its associated PDF represents the intra-subject distances and the dark grey histogram and associated PDF the inter-subject distances. In the case of all histograms, the range of values has been divided into 10 bins.

The histograms for the temporal domain shown in Figure 9 can be seen by inspection to have improved separation and lower overlap between the intra- and inter-subject distributions, when compared with the cepstral domain data shown in Figure 10, which 
exhibits higher overlap, corresponding to the lower performance of the biometric identification algorithm used when applied to the cepstral domain.

Figure 9 Histograms and fitted parametric distributions of transverse adult TEOAE corpus in temporal domain (light grey is intradistances, dark grey is interdistances)

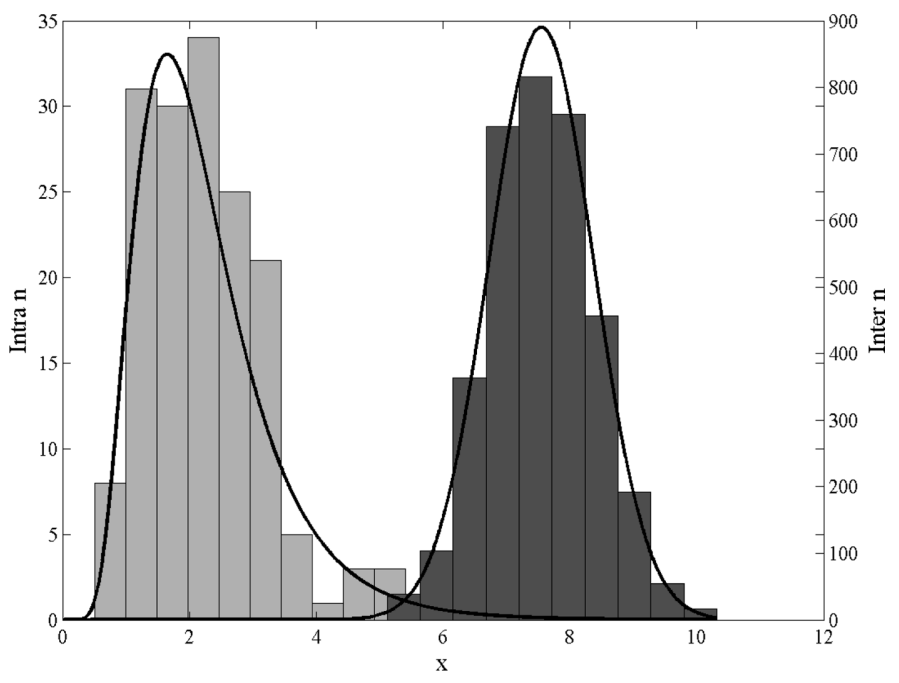

Figure 10 Histograms and fitted parametric distributions of transverse adult TEOAE corpus in cepstral domain (light grey is intradistances, dark grey is interdistances)

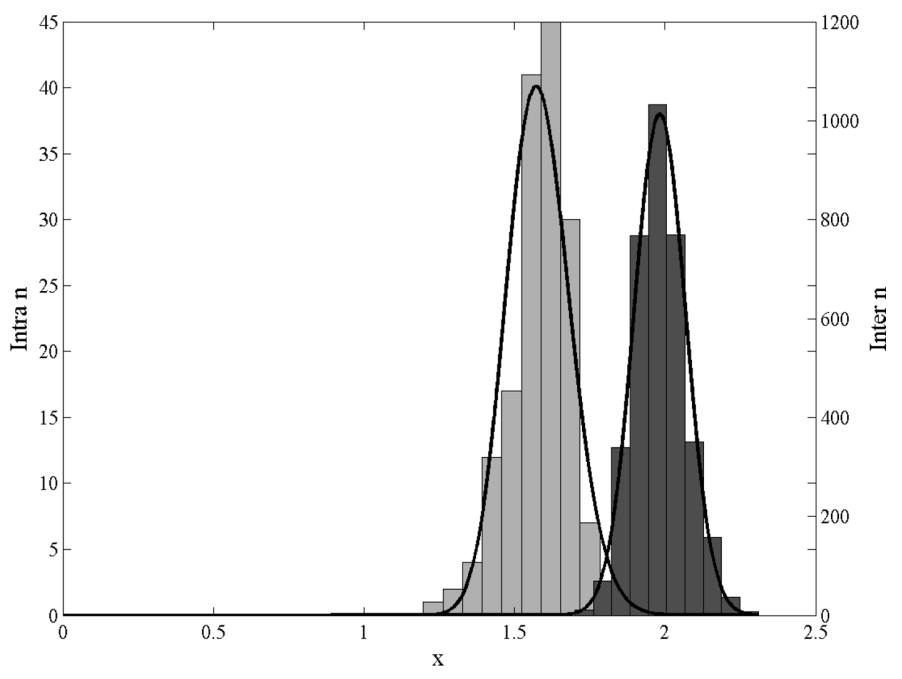

\section{Conclusions}

This paper has defined the properties of TEOAEs that make them suitable for use as a biometric; some example TEOAEs have been shown and can be seen to differ between individuals. Metrics used to compare and quantify the operation of biometric systems, namely the FAR, the FRR and the EER, have been defined. A basic analysis using 
area-under-the-curve applied to the TEOAE recordings was used to show difference between individuals, but repeatability within one individual. A Euclidean distance analysis has been applied to a sample of five individuals and shown to be able to distinguish between different individuals. The concept of the cepstrum transform and cepstral domain has been introduced and applied to the existing temporal TEOAE recordings. The same Euclidean analysis was applied to the cepstral domain data as used for the temporal tests. Determination of an individual's identification was seen to be possible using this technique. To compare the performance of the Euclidean distance measure in both the temporal and the cepstral domains, a further data set of recorded TEOAEs was utilised, consisting of a larger number of individuals than the data set used with the earlier analysis. The process used to perform the analysis was detailed, as were the biometric and statistical parameters used in the comparison. In addition to the numerical results to aid in comparison, histograms and fitted parametric distributions were presented for both operational domains.

This paper has shown that when using a Euclidean distance technique it can achieve better performance when applied to the TEOAE data when in the temporal domain, as opposed to in the cepstral domain. Operation in the cepstral domain may still prove desirable for certain applications as removal of cepstral coefficients after performing the cepstrum transform may allow masking-off or removal of noise artefacts from the recorded TEOAE. The use of the cepstrum transformation applied to biometric feature extraction has been demonstrated and critically compared with the existing techniques.

A potential route to improving the operation of the Euclidean distance approach would be to apply it to the response in the frequency domain, this would allow selection of frequency bands of interest and permit the reduction of the influence of noise in the resulting biometric identity as frequencies out of the range(s) of interest can be readily disregarded. The efficiency of the cepstrum approach is limited by the absence of harmonically generated components due to the propagation characteristics of the cochlea. Any resulting periodicity detected by the cepstrum process will be co-incidental but should remain stable for the subject in question, though the lack of systematically generated periodicity in the frequency response will lead to a less-rich biometric than would be achieved if there was periodicity in the response attributable to the structure of the subject's cochlea.

\section{Acknowledgements}

The authors wish to gratefully acknowledge the assistance provided by the test subjects from whom the TEOAE recordings were made, and also the support of the UK Engineering and Physical Sciences Research Council for their support for this work under grant EP/E015522/1.

\section{References}

Bilger, R.C., Matthies, M.L., Hammel, D.R. and Demorest, M.E. (1990) 'Genetic-implications of gender differences in the prevalence of spontaneous otoacoustic emissions', Journal of Speech and Hearing Research, Vol. 33, No. 3, pp.418-432, Available from: ISI:A1990DY14700001. 
Charbuillet, C., Gas, B., Chetouani, M. and Zarader, J.L. (2009) 'Optimizing feature complementarity by evolution strategy: application to automatic speaker verification', Speech Communication, Vol. 51, No. 9, pp.724-731, Available from: http://www.sciencedirect.com/ science/article/B6V1C-4VH4D8W-1/2/961a43b26aa0fc8ea854c57722f7b928

Cope, Y. and Lutman, M.E. (1988) 'Otoacoustic emissions', in McCormick, B. (Ed.): Paediatric Audiology 0-5 years, Taylor \& Francis, London, pp.221-245.

Doddington, G.R., Przybocki, M.A., Martin, A.F. and Reynolds, D.A. (2000) 'The NIST speaker recognition evaluation - overview, methodology, systems, results, perspective', Speech Communication, Vol. 31, Nos. 2-3, pp.225-254, Available from: http://www.sciencedirect. com/science/article/B6V1C-40D61JP-D/2/50569b7e672831d5317b597943c28be2

Gold, T. (1948) 'Hearing. II. The physical basis of the action of the cochlea', Proceedings of the Royal Society of London Series B, Biological Sciences, Vol. 135, No. 881, pp.492-498, Available from: http://www.jstor.org/stable/82559

Grandori, F. and Ravazzani, P. (1993) 'Non-linearities of click-evoked otoacoustic emissions and the derived non-linear technique', British Journal of Audiology, Vol. 27, No. 2, pp.97-102. Available from: http://www.informaworld.com/10.3109/03005369309077898

Kemp, D.T. (1978) 'Stimulated acoustic emissions from within the human auditory-system', Journal of the Acoustical Society of America, Vol. 64, No. 5, pp.1386-1391, Available from: ISI: A1978FW33500015.

Knight, R.D. and Kemp, D.T. (2000) 'Indications of different distortion product otoacoustic emission mechanisms from a detailed $\mathrm{f}(1), \mathrm{f}(2)$ area study', Journal of the Acoustical Society of America, Vol. 107, No. 1, pp.457-473, Available from: ISI:000085225900039.

Lutman, M.E., Davis, A.C., Fortnum, H.M. and Wood, S. (1997) 'Field sensitivity of targeted neonatal hearing screening by transient-evoked otoacoustic emissions', Ear and Hearing, Vol. 18, No. 4, pp.265-276, Available from: ISI:A1997XU40600002.

Mansfield, A.J. and Wayman, J.L. (2002) Best Practices in Testing and Reporting Performance of Biometric Devices, National Physical Laboratory Queens Road, Teddington, Middlesex, TW11 0LW, CMSC 14/02.

Oppenheim, A.V. and Schafer, R.W. (2004) 'From frequency to quefrency: a history of the cepstrum', IEEE Signal Processing Magazine, Vol. 21, No. 5, pp.95-99, Available from ISI: 000223480700013.

Robinette, M.S. and Glattke, T.J. (1997) Otoacoustic Emissions: Clinical Applications, 3rd ed., Thieme, New York

Severini, T.A. (2000) Likelihood Methods in Statistics, T.A. Severini, Oxford University Press, Oxford, UK, ISBN 0-19-850650-3.

Swabey, M.A., Beeby, S.P., Brown, A.D. and Chad, J.E. (2004) 'Using otoacoustic emissions as a biometric', Biometric Authentication, Proceedings, Vol. 3072, pp.600-606, Available from: ISI:000222868800082.

Swabey, M.A., Chambers, P., Lutman, M.E., White, N.M., Chad, J.E., Brown, A.D. and Beeby, S.P. (2009) 'The biometric potential of transient otoacoustic emissions', International Journal of Biometrics (IJBM), Vol. 1, No. 3, pp.349-364, Available from: http: //eprints.ecs. soton.ac.uk/17295/

Vohr, B.R., Carty, L.M., Moore, P.E. and Letourneau, K. (1998) 'The rhode island hearing assessment program: experience with statewide hearing screening (1993-1996)', Journal of Pediatrics, Vol. 133, No. 3, pp.353-357, Available from: ISI:000075772900011.

Whitehead, M.L., Kamal, N., Lonsburymartin, B.L. and Martin, G.K. (1993) 'Spontaneous otoacoustic emissions in different racial groups', Scandinavian Audiology, Vol. 22, No. 1, pp.3-10, Available from: ISI:A1993KM96000001. 